\title{
Twelve-Year Longitudinal Trends in Trachoma Prevalence among Children Aged 1-9 Years in Amhara, Ethiopia, 2007-2019
}

\author{
Eshetu Sata, ${ }^{1}$ Andrew W. Nute, ${ }^{2}$ Tigist Astale, ${ }^{1}$ Demelash Gessese, ${ }^{1}$ Zebene Ayele, ${ }^{1}$ Mulat Zerihun, ${ }^{1}$ Ambahun Chernet, ${ }^{1}$ \\ Berhanu Melak, ${ }^{1}$ Kimberly A. Jensen, ${ }^{2}$ Mahteme Haile, ${ }^{3}$ Taye Zeru, ${ }^{3}$ Melkamu Beyen, ${ }^{4}$ Adisu Abebe Dawed, ${ }^{4}$ Fikre Seife, ${ }^{5}$ \\ Zerihun Tadesse, ${ }^{1}$ Elizabeth Kelly Callahan, ${ }^{2}$ Jeremiah Ngondi, ${ }^{6}$ and Scott D Nash ${ }^{2 \star}$ \\ ${ }^{1}$ Trachoma Control Program, The Carter Center, Addis Ababa, Ethiopia; ${ }^{2}$ Trachoma Control Program, The Carter Center, Atlanta, Georgia; \\ ${ }^{3}$ Amhara Public Health Institute, Research and Technology Transfer Directorate, Bahir Dar, Ethiopia; ${ }^{4}$ Amhara Regional Health Bureau, Health \\ Promotion and Disease Prevention, Bahir Dar, Ethiopia; ${ }^{5}$ Federal Ministry of Health, Disease Prevention and Control Directorate, Addis Ababa, \\ Ethiopia; ${ }^{6}$ RTI International, International Development (Global Health), London, United Kingdom
}

\begin{abstract}
Trachoma control in the Amhara region of Ethiopia, where all districts were once endemic, began in 2001 and attained full scale-up of the Surgery, Antibiotics, Facial cleanliness, and Environmental improvement (SAFE) strategy by 2010. Since scaling up, the program has distributed approximately 14 million doses of antibiotic per year, implemented village- and school-based health education, and promoted latrine construction. This report aims to provide an update on the prevalence of trachoma among children aged 1-9 years as of the most recent impact or surveillance survey in all 160 districts of Amhara. As of 2019, 45 (28\%) districts had a trachomatous inflammation-follicular (TF) prevalence below the $5 \%$ elimination threshold. There was a statistically significant relationship between TF prevalence observed at the first impact survey (2010-2015) and eventual achievement of TF < 5\% (2015-2019). Of the 26 districts with a first impact survey $<10 \%$ TF, $20(76.9 \%)$ had $<5 \%$ TF at the most recent survey. Of the 75 districts with a first survey between $10 \%$ and $29.9 \% \mathrm{TF}, 21(28.0 \%)$ had $<5 \%$ TF at the most recent survey. Finally, among 59 districts $\geq 30 \%$ TF at the first survey, four $(6.8 \%)$ had $<5 \%$ TF by 2019 . As of 2019,30 (18.8\%) districts remained with TF $\geq 30 \%$. Amhara has seen considerable reductions of trachoma since the start of the program. A strong commitment to the SAFE strategy coupled with datadriven enhancements to that strategy is necessary to facilitate timely elimination of trachoma as a public health problem regionally in Amhara and nationwide in Ethiopia.
\end{abstract}

\section{INTRODUCTION}

To eliminate trachoma as a public health problem, the WHO recommends a four-part strategy, surgery for correcting trachomatous trichiasis (TT), antibiotics to clear Chlamydia trachomatis $(\mathrm{Ct})$ infection, and facial cleanliness and environmental improvement to reduce transmission (Surgery, Antibiotics, Facial cleanliness, and Environmental improvement [SAFE]). The SAFE strategy has continually shown to be effective in reducing trachoma to below elimination thresholds, especially in countries with low-tomoderate trachoma endemicity. ${ }^{1}$ In 1996 , the WHO established the WHO Alliance for the Global Elimination of Trachoma by the year 2020 (GET2020). ${ }^{2}$ Although the global elimination of trachoma as a public health problem by 2020 was not achieved, the number of people at risk for trachoma has been reduced by $91 \%$ since $2002 .{ }^{1}$ Furthermore, as of 2020,10 countries have been validated by the WHO to have eliminated trachoma as a public health problem. ${ }^{1}$ With these successes in mind, a new trachoma elimination target date of 2030 may be within reach.

The Amhara National Regional State is a mountainous region in northwest Ethiopia, with a population of approximately 22 million people. Historically, Amhara has had one of the highest burdens of trachoma in the world. ${ }^{3-9}$ Before the start of SAFE interventions, a prevalence of trachomatous inflammation-follicular (TF) as high as $90 \%$ and $C t$ infection as high as $50 \%$ were recorded within the region. ${ }^{8-10}$ A 2007 region-wide baseline survey provided overwhelming evidence that trachoma was a serious public health problem in all areas

*Address correspondence to Scott D. Nash, The Carter Center, Trachoma Control Program, 453 John Lewis Freedom Parkway NE, Atlanta, GA 30307. E-mail: scott.nash@cartercenter.org of Amhara, and therefore, SAFE was scaled up between 2007 and 2010 to cover the entire region. ${ }^{11}$

The Trachoma Control Program has implemented the full SAFE strategy in Amhara from scale-up through 2019. Throughout this time period, considerable programmatic efforts were undertaken, and progress has been made on reducing the burden of trachoma. ${ }^{8,12-15}$ However, evidence is mounting that in some parts of the region, trachoma continues to persist among children, despite $A, F$, and $E$ interventions. ${ }^{15-18}$ Persistent trachoma may yet be identified throughout the country as other regions in Ethiopia, which scaled up after Amhara, begin to assess the impact of $A, F$, and $E$ in a wider manner. Globally, $46 \%$ of individuals living in trachoma-endemic districts (TF $\geq 5 \%$ ) are in Ethiopia, and therefore, if global elimination of trachoma as a public health problem is to be achieved, it is imperative to understand how trachoma responds to the SAFE strategy in Ethiopia over the long term. ${ }^{1}$

The aim of this report was to comprehensively examine the trends in active trachoma, TF, and trachomatous inflammationintense (TI), among children aged 1-9 years over a 12-year period in Amhara. Further data on levels of water, sanitation, and hygiene (WASH), as well as post-endemic surveillance activities were also highlighted.

\section{METHODS}

Ethical considerations. Survey methodology for all surveys was approved by the Ethical Review Committee of the Amhara Regional Health Bureau, Ethiopia, and Emory University Institutional Review Board under protocol 079-2006. Permission to obtain verbal informed consent and verbal assent was granted by the review boards due to the high rate of illiteracy among the study population. For surveys conducted 
between 2017 and 2019, survey protocols were also reviewed by Tropical Data (https://www.tropicaldata.org/). Verbal informed consent to conduct a household interview was obtained from the head of each household or a representative aged $\geq 18$ years. Verbal informed consent and assent were obtained from all individuals examined for trachoma and recorded on the household questionnaire.

Time line and setting. The Trachoma Control Program began in four pilot districts in central Amhara in 2001 and was expanded to cover the entire region by $2010 .^{8}$ From 2010 until 2019, the SAFE strategy was implemented throughout the entire region of Amhara. ${ }^{11,14,15}$ Between 2010 and 2015, the first full set of impact surveys was conducted to measure the impact of the SAFE strategy on trachoma prevalence in the region. Those surveys demonstrated that 143/152 (94\%) districts remained endemic $(\geq 5 \%)$ for TF. ${ }^{15}$ Stewart et al. ${ }^{15}$ also reported the considerable SAFE inputs implemented by the program throughout the region during that time period.

Between 2016 and 2019, SAFE interventions continued throughout the region (Table 1). Approximately 14 million doses of antibiotic were delivered annually (Supplemental Figure 1), and administrative reports of programmatic antibiotic coverage were close to $90 \%$ each year (Supplemental Figure 2). Population-based coverage evaluation surveys conducted during this period demonstrated self-reported coverage to be $76.8 \%$ region-wide, with estimates ranging between $78.5 \%$ and $86.9 \%$ in select hyperendemic districts. ${ }^{19,20}$ A school trachoma program (STP), created in 2015, enrolled approximately 8,000 schools region-wide per year, trained more than 17,000 teachers in trachoma curricula, and assessed more than 500,000 school children for clean faces. ${ }^{21}$ Other $F$ and $E$ interventions implemented during this time included the training of hygiene and environmental health officers, advocating for new latrine construction, and the tracking of newly constructed latrines. The region has also established a WASH-neglected tropical disease (NTD) technical working group and task force to enhance collaboration between trachoma/NTD and WASH sectors.
Survey methodology. Baseline survey 2007. A baseline survey was conducted throughout Amhara in 2007 to determine the prevalence of TF among children aged 1-9 years at the zonal level as described previously by Emerson et al. ${ }^{11}$ Conducting a baseline survey at an evaluation unit larger than the district is an acceptable practice in the trachoma field. ${ }^{22}$ Baseline zonal estimates were provided in this report not for direct comparison with district estimates but to provide context for starting SAFE interventions.

Impact and surveillance surveys, 2010-2017. The methodology for the trachoma impact and surveillance surveys conducted between 2010 and 2017 have previously been described extensively. ${ }^{14-16,18,23}$ These surveys were conducted to estimate the impact of 3-8 years of the SAFE strategy on the prevalence of TF among children aged 1-9 years. Although the methodology has evolved over time, all surveys in this time frame were population-based and used a multistage cluster sampling approach.

Impact and surveillance surveys, 2017-2019. Beginning in 2017, the Federal Ministry of Health of Ethiopia began using the Tropical Data System for trachoma surveys throughout the country. The methodology for these surveys has been published previously and is similar to what was historically used in Amhara. ${ }^{24}$ This methodology called for a multistage design, whereby in the first stage of sampling, villages were chosen either by a random selection or chosen using a probability proportional to estimated size, and in the second stage, one development team of approximately 30 households was randomly chosen. All individuals aged $\geq 1$ year in all households in each selected segment were enumerated, and present, consenting individuals were examined for the signs of trachoma. The sample size for surveys conducted during this time was the same, regardless of the likelihood of a TF prevalence reaching elimination thresholds in a district. With the assumption of $4 \% \mathrm{TF} \pm 2 \%$ and a design effect ranging from 2.63 to 2.71 , surveys were designed to examine approximately 1,200 children per district. Given the demographics in Amhara, to attain the required sample size, 30 clusters of 30 households were sampled in nearly all districts.

TABLE 1

Summary of regional AFE activities implemented for trachoma control, Amhara, Ethiopia, 2016-2019

\begin{tabular}{|c|c|c|c|c|}
\hline Activity & 2016 & 2017 & 2018 & 2019 \\
\hline $\begin{array}{l}\text { Number of people treated with } \\
\text { azithromycin }^{\circledR}\end{array}$ & $15,016,928$ & $14,248,325$ & $13,650,639$ & $14,138,710$ \\
\hline $\begin{array}{l}\text { Number of people treated with } \\
\text { tetracycline eye ointment }\end{array}$ & 323,218 & 305,358 & 304,430 & 325,341 \\
\hline $\begin{array}{l}\text { Median number of cumulative MDA } \\
\text { rounds }\end{array}$ & 8 (IQR: 8-9) & 9 (IQR: 8-10) & 10 (IQR: 9-11) & 11 (IQR: 9-11) \\
\hline Antibiotic coverage ${ }^{\star}$ & $94.5 \%$ (SD: $4.2 \%)$ & $91.7 \%$ (SD: $7.0 \%)$ & 89.3\% (SD: 8.3\%) & 89.3\% (SD: 8.7\%) \\
\hline School teachers trained for STP† & 3,468 & 5,092 & - & 8,709 \\
\hline Schools enrolled in STP & 8,384 & 8,384 & 8,465 & 8,677 \\
\hline $\begin{array}{l}\text { School children assessed for facial } \\
\text { cleanliness }\end{array}$ & 46,104 & 160,605 & 200,025 & 149,545 \\
\hline Clean face school children & $92.4 \%$ & $90.1 \%$ & $89.0 \%$ & $89.0 \%$ \\
\hline $\begin{array}{l}\text { Village children assessed for facial } \\
\text { cleanliness }\end{array}$ & 6,050 & 16,714 & 15,360 & 13,504 \\
\hline Clean face village children & $77.0 \%$ & $77.0 \%$ & $79.0 \%$ & $75.0 \%$ \\
\hline $\begin{array}{l}\text { Trained hygiene and environmental } \\
\text { health officers }\end{array}$ & - & 292 & - & 368 \\
\hline New household latrines & 484,475 & 111,018 & 872,801 & 495,155 \\
\hline
\end{tabular}


Training. The same grader training methods were used for all surveys conducted between 2010 and 2017 and have been described elsewhere. ${ }^{15,16,23}$ The grader trainings conducted between 2017 and 2019 were similar to those conducted previously, both in content of the trainings and inter-grader assessments (IGAs). Between 2017 and 2019, grader trainees were trained to identify TF, TI, and TT. Graders who passed the slide-based IGAs subsequently undertook a field-based IGA by examining 50 children. To qualify, grader trainees needed to score a kappa of at least 0.7 for the sign TF, when compared with a single "grader trainer" who had previously passed training for trainers given by a Tropical Data certified master trainer. ${ }^{24}$ In addition, graders were required to have passed Tropical Data training and/or participated in a survey within the prior 12 months to be eligible to participate in survey fieldwork.

Before each survey, data recorders participated in an approximately 3-day training on how to use the survey software, which included classroom and field-based components. Training also included instruction on the consent process and how to properly segment a village and concluded with a recorder examination.

Data collection. For all surveys, recorders entered data electronically using Open Data Kit Applications (Get ODK, github.com/getodk), either using Swift Insights software on electronic tablets (2011-2017) or on a Tropical Data app loaded on Android phones (2017-2019). ${ }^{14,15,24,25}$ All surveys included a household-level questionnaire which contained WASH indicators. Analysis of WASH indicators was based on WHO/ UNICEF Joint Monitoring Program definitions for improved and unimproved water sources and sanitation facilities. ${ }^{26}$ Distance to water was defined as the time it takes to retrieve water for any use and return home. ${ }^{15,24}$ The presence of a clean face was determined by graders as those children aged 1-9 years with neither ocular nor nasal discharge. The clean face variable was not part of the standard survey after 2017, so clean face data for these surveys were collected in the "Notes" field using a simple code covered in training. Clean face data were missing for five districts in the 2017 survey.

In all surveys from 2010 through 2019, certified graders used $\times 2.5$ magnification loupes and appropriate lighting to examine eligible individuals for signs of trachoma using the WHO simplified grading system. ${ }^{27}$ Identified cases of active trachoma (TF and/or $\mathrm{TI}$ ) were offered treatment with $1 \%$ tetracycline eye ointment to be used twice daily for 6 weeks, according to current WHO guidelines, and identified cases of TT were referred to the nearest health post for surgical services.

Statistical analysis. Analysis techniques for the surveys conducted between 2010 and 2017 have been detailed previously. ${ }^{11,14,15,23}$ In brief, estimates were calculated with Stata (Stata Corporation, College Station, TX) survey commands using weights calculated as the inverse of the sampling probability of both stages of sampling. Robust Cls were estimated using Taylor series linearization which accounted for levels of clustering (cluster and household). Starting in 2017, TF was estimated using an algorithm which first age-adjusted cluster-level data in 1-year age bands using the 2007 Ethiopian National Census population data. ${ }^{24}$ The mean of the cluster prevalence estimates was used as the district prevalence. A previously described bootstrap method was used to estimate Cls for those estimates. ${ }^{24}$ District TI and clean face prevalence estimates and Cls were calculated in a manner similar to TF. $R$ and corresponding survey package ( $R$ Core Team 2013) were used to account for the clustered nature of the data when calculating estimates and robust Cls of agespecific prevalence and corresponding age-trend tests. ${ }^{28} \mathrm{~A}$ trend test was used to compare the proportion of districts which reached the $<5 \%$ threshold by the first impact survey WHO TF prevalence category and the proportion of districts which remained $<5 \%$ at surveillance survey by the first impact survey WHO TF prevalence category using the Stata "ptrend" command. Maps were created using ArcGIS Pro v. 2.6.2 (Esri, Redlands, CA) to customize and map shapefiles available on the Humanitarian Data Exchange (https://data.humdata.org) through the UN Office of Coordination of Humanitarian Affairs.

Between 2016 and 2019, the district count changed in Amhara from 152 to 160 because of administrative splitting of some districts. When two or more districts were split from a parent district, they were each assigned the parent district prevalence until a new survey was conducted. Two districts were formed in 2018 from three parent districts each, and for these districts, no prior prevalence was assigned. Five districts had not received a second survey as of 2019, and therefore, their first survey estimate was carried forward for this analysis. District TF prevalence estimates were compared over time to determine which had a percent decrease $\geq 20 \%$, an arbitrary cut-point but one that is likely programmatically relevant. Because programmatic thresholds have not been developed for $\mathrm{TI}$, we used a TI prevalence of $3 \%$ as an arbitrary threshold.

\section{RESULTS}

Between 2015 and 2019, 160 population-based surveys were conducted which covered all 10 zones in Amhara (Table 2). These surveys sampled 131,514 households across 4,096 clusters and enumerated 127,456 children aged 1-9 years, of whom 124,249 (97.5\%) were examined for trachoma. The median number of years of $A, F$, and $E$ interventions per district before these surveys was nine.

Based on the household-level WASH questionnaire, median (interquartile range [IQR]) prevalence of key indicators, by district, were as follows: $39.3 \%$ (28.0-50.4\%) of households had access to water within 30 minutes, $61.6 \%$ (49.5-73.5\%) of households had an improved water source, and $2.9 \%$ (0.8-11.6\%) of households had improved sanitation (Figure 1). Among children aged 1-9 years, the median (IQR) prevalence of directly observed clean face was $69.7 \%$ (64.6-76.8\%).

There was a statistically significant correlation between the TF prevalence observed at the first impact survey (2010-2015) and eventual achievement of the TF elimination threshold of $<5 \%$. Of the 160 districts, $20 / 26$ (76.9\%) districts with $<10 \%$ TF at the first survey had $<5 \%$ TF at most recent survey (Figure 2), 21/75 (28.0\%) districts with between $10 \%$ and $29.9 \%$ TF at the first survey had $<5 \%$ TF at most recent survey (Figure 3), and only 4/59 (6.8\%) districts with $\geq 30 \%$ TF at the first survey had reached $<5 \%$ TF at most recent survey (Figure 4) (Ptrend $<0.001$ ). As of 2019, 30 (18.8\%) districts remained with TF $\geq 30 \%$ and ranged in TF prevalence from $30.0 \%$ to $55.3 \%$.

As of 2019, the district TI prevalence among children aged $1-9$ years ranged from $0.0 \%$ to $15.9 \%$ (Figure 5 ). In the region as a whole, the TI prevalence was < $3 \%$ in 129/160 (80.6\%) districts. The zones with the highest percentage of districts 
TABLE 2

Sample size for the 160 most recent surveys encompassing Amhara, Ethiopia, conducted between 2015 and 2019

\begin{tabular}{|c|c|c|c|c|c|c|c|}
\hline \multirow[b]{2}{*}{ Zone } & \multirow[b]{2}{*}{ Districts } & \multirow[b]{2}{*}{ Clusters } & \multirow[b]{2}{*}{ Households } & \multicolumn{2}{|c|}{ Enumerated } & \multicolumn{2}{|c|}{ Examined } \\
\hline & & & & All ages & Children (1-9 years) & All ages & Children (1-9 years) \\
\hline Awi & 12 & 346 & 11,021 & 46,021 & 11,429 & 38,868 & 11,235 \\
\hline East Gojam & 19 & 570 & 17,486 & 67,052 & 14,470 & 57,193 & 14,214 \\
\hline North Gondar & 24 & 588 & 19,630 & 80,416 & 22,746 & 66,040 & 22,060 \\
\hline North Shoa & 24 & 616 & 20,898 & 78,430 & 17,299 & 65,678 & 16,806 \\
\hline North Wollo & 14 & 420 & 13,211 & 48,174 & 11,710 & 40,408 & 11,463 \\
\hline Oromia & 7 & 210 & 6,312 & 25,488 & 7,914 & 21,581 & 7,718 \\
\hline South Gondar & 13 & 324 & 10,009 & 38,368 & 9,765 & 32,033 & 9,499 \\
\hline South Wollo & 22 & 440 & 14,208 & 53,026 & 11,889 & 43,699 & 11,506 \\
\hline Waghemra & 8 & 174 & 5,403 & 21,694 & 6,663 & 18,408 & 6,483 \\
\hline West Gojam & 17 & 408 & 13,336 & 54,255 & 13,571 & 45,133 & 13,265 \\
\hline Amhara region & 160 & 4,096 & 131,514 & 512,924 & 127,456 & 429,041 & 124,249 \\
\hline
\end{tabular}

with $\mathrm{TI} \geq 3 \%$ were Waghemra (37.5\%) and South Gondar (38.5\%) zones, whereas Awi and Oromia zones had 0 districts still $\geq 3 \%$.

As of these current surveys, $37 / 160(23.1 \%)$ districts had reached the TF elimination threshold and had completed surveillance surveys. Among these 37 districts, 28 (75.7\%) remained below the $5 \%$ TF threshold at surveillance survey (Figure 6). There was a statistically significant correlation between the TF prevalence observed at the first impact survey and the prevalence at surveillance survey. Of 37 districts with a surveillance survey, all seven districts with a first impact survey $<5 \%$ TF remained $<5 \%$ at surveillance, 11 districts with a first impact survey between $5 \%$ and $9.9 \%$ TF, $9(81.8 \%)$ remained $<5 \%$ at surveillance; and 19 districts with a first impact survey between $10 \%$ and $29.9 \%, 12(63.2 \%)$ remained $<5 \%$ (Ptrend $=0.04$ ). Among the nine districts where TF was $\geq 5 \%$ at surveillance, the TF prevalence at surveillance ranged from $5.1 \%(95 \% \mathrm{Cl}$ : 3.2-7.4) to $11.2 \%$ (95\% Cl: 4.6-18.2). The prevalence of $\mathrm{TI}$ was $\leq 1 \%$ in all nine districts.

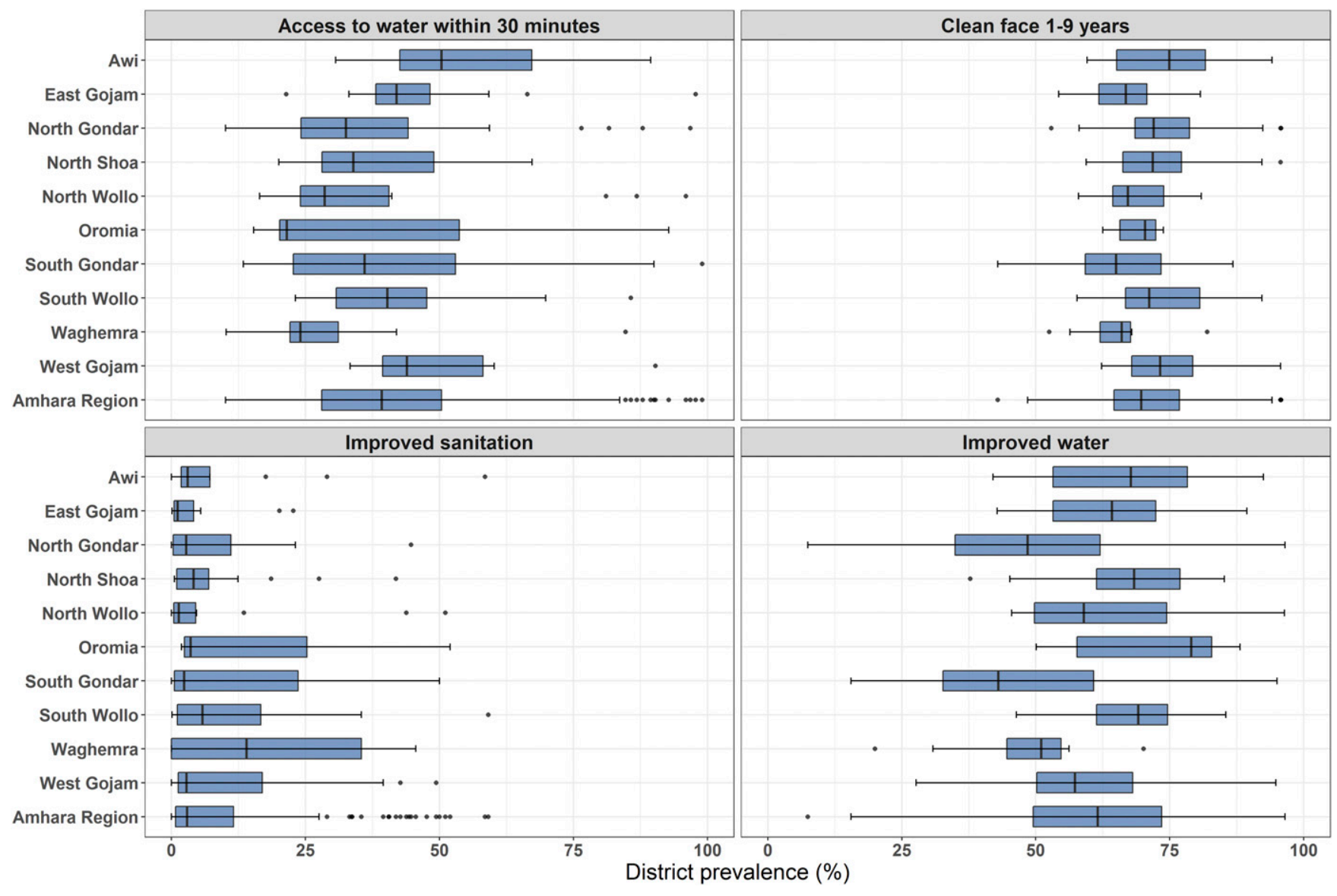

FIGURE 1. Prevalence distribution of water, sanitation, and hygiene indicators by zone, Amhara, Ethiopia, 2015-2019. Figures show the median, first and third quartiles, range, and outliers. ${ }^{*}$ Clean face and sanitation were directly observed. Improved sanitation defined as at least a pit latrine with at least a slab of concrete; improved water defined as one of the following: protected spring, hand pump/tube well/borehole, public piped water/tap/ standpipe, private piped into yard/dwelling, or rainwater collection. This figure appears in color at www.ajtmh.org. 


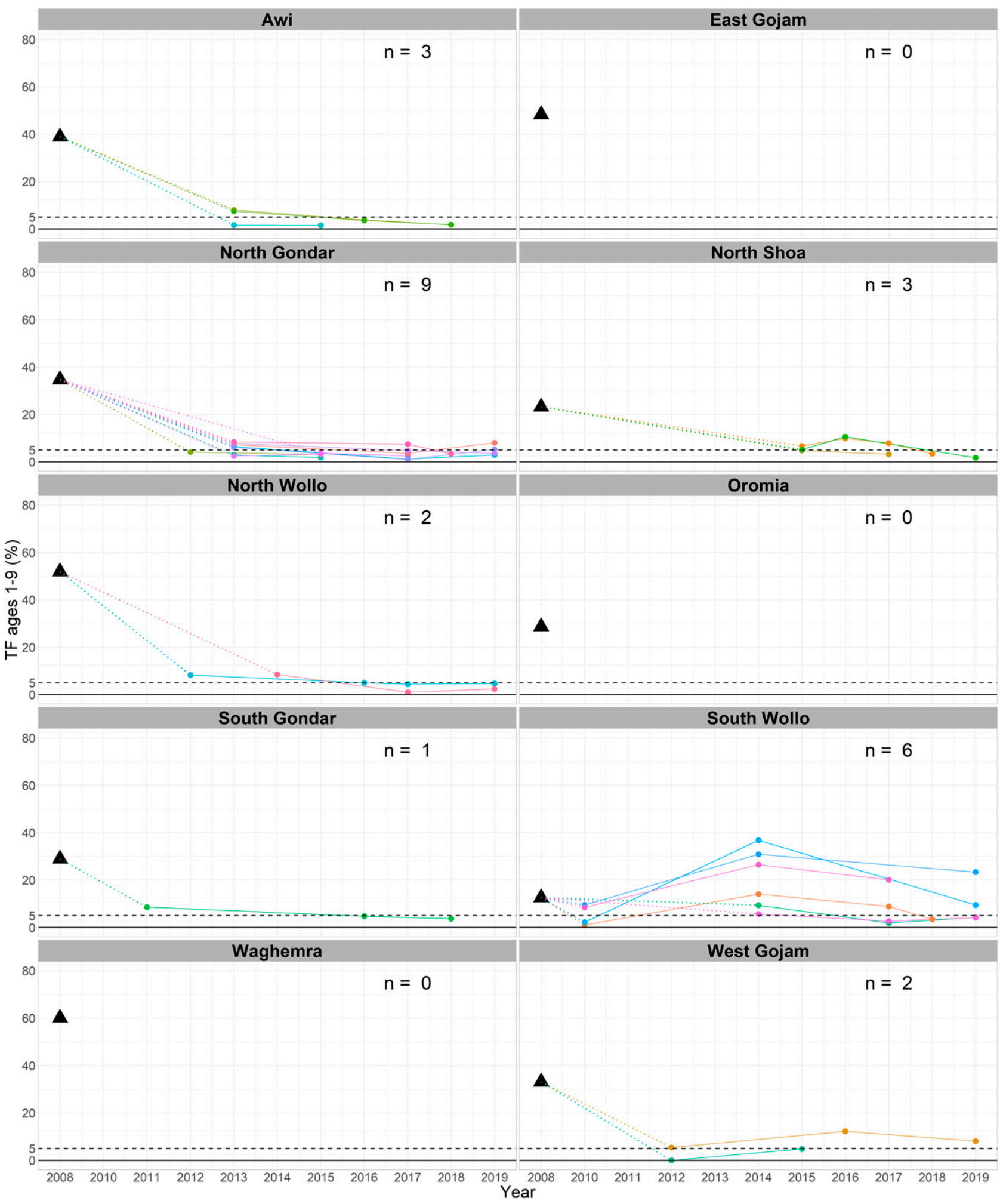

FIGURE 2. Trachomatous inflammation-follicular (TF) prevalence over time among districts with a first impact survey at prevalence less than $10 \%$ among children aged 1-9 years, Amhara, Ethiopia, 2007-2019 $(n=26)$. Triangles represent zonal-level baseline estimates. This figure appears in color at www.ajtmh.org.

The age-specific TF prevalence among children aged 1-9 years in the current survey was lower for each year of age than that among children surveyed in 2010-2015 (Figure 7A). In the current surveys, the prevalence of TF among older children was considerably lower (Ptrend $<0.001$ ) than those of younger children. The age-specific prevalence of TI was also consistently lower in the current surveys than that in children surveyed in 2010-2015 (Figure 7B). The current TI prevalence ranged between $0.6 \%$ among children aged 8 and 9 years to $3.0 \%$ and $2.7 \%$ among children aged 1 and 2 years, respectively. 


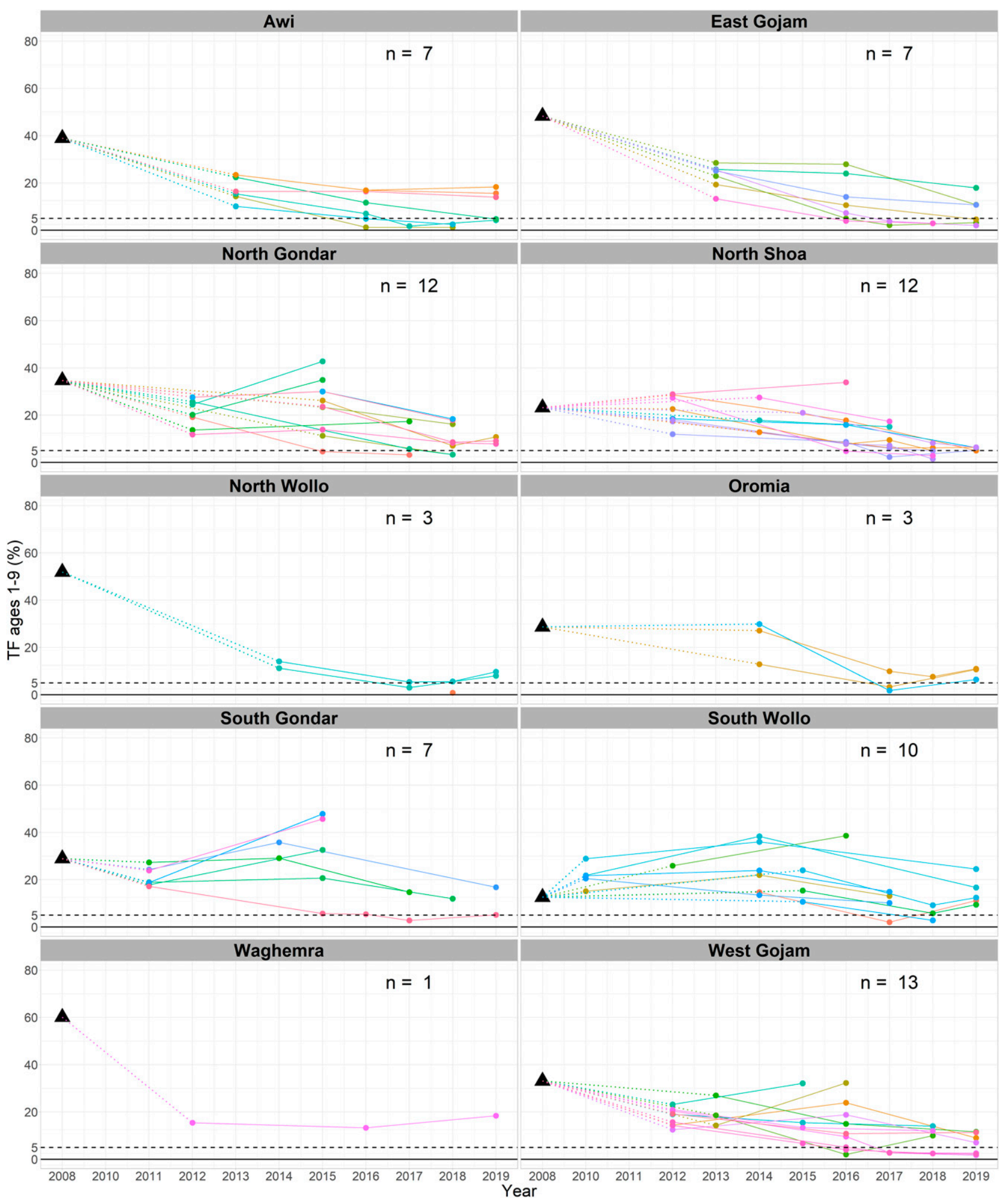

FIGURE 3. Trachomatous inflammation-follicular (TF) prevalence over time among districts with a first impact survey at prevalence between $10 \%$ and $29.9 \%$ among children aged $1-9$ years, Amhara, Ethiopia, 2007-2019 $(n=75)$. Triangles represent zonal-level baseline estimates. This figure appears in color at www.ajtmh.org.

As of 2019, all 10 zones had at least one district still endemic for trachoma (Supplemental Figure 3). The highest concentration of districts with a TF prevalence $<5 \%$, or between $5 \%$ and $9.9 \%$, were in Awi (66.7\%), North Gondar (58.3\%), or North Shoa (62.5\%) zones (Supplemental Table 1). The highest concentration of districts which remained $\geq 30 \%$ TF were in South Gondar (46.2\%) and Waghemra $(50.1 \%)$ zones located in the central and northeastern part of Amhara. Region-wide, when comparing between the 2010-2015 and the 2016-2019 surveys, 115/160 (71.9\%) districts have seen a $\geq 20 \%$ decrease in TF prevalence. Based on the WHOTF prevalence 


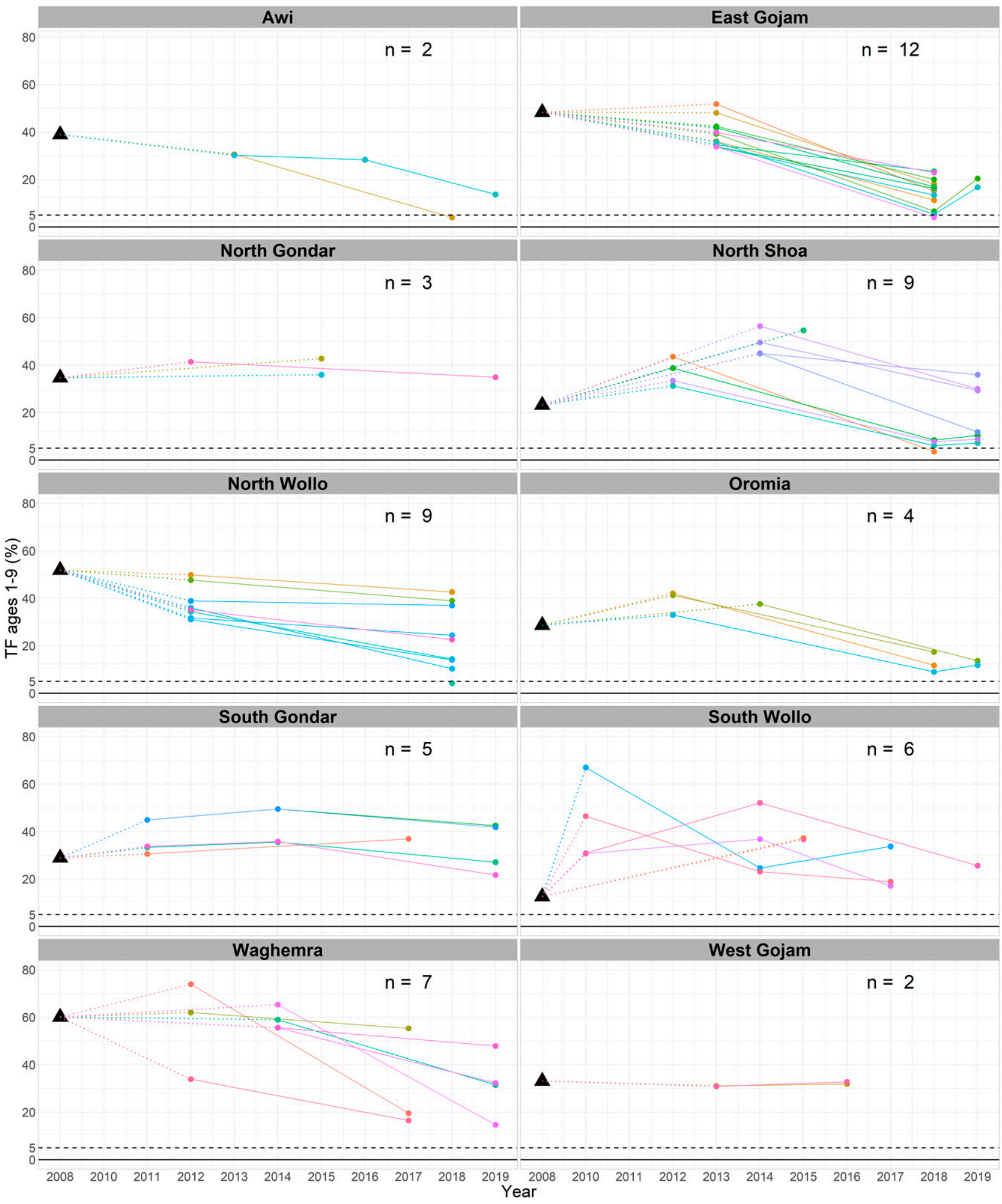

FIGURE 4. Trachomatous inflammation-follicular (TF) prevalence over time among districts with a first impact survey at prevalence greater than or equal to $30 \%$ among children aged $1-9$ years, Amhara, Ethiopia, 2007-2019 $(n=59)$. Triangles represent zonal-level baseline estimates. This figure appears in color at www.ajtmh.org.

categories, the number of districts with a TF prevalence $<5 \%$ has increased from $9 / 152$ (5.9\%) districts as of 2015 to $45 / 160(28.1 \%)$ districts as of 2019 (Supplemental Figure 4). Furthermore, the number of districts with a TF prevalence $\geq 30 \%$ has decreased from $67 / 152(44.1 \%)$ as of 2015 to $30 / 160(18.8 \%)$ as of 2019.

\section{DISCUSSION}

Substantial reductions have been observed in the prevalence of TF over a 12-year period in Amhara, Ethiopia. As of $2019,72 \%$ of districts had seen $a \geq 20 \%$ reduction in TF prevalence, and $28 \%$ of districts had reached the threshold for 


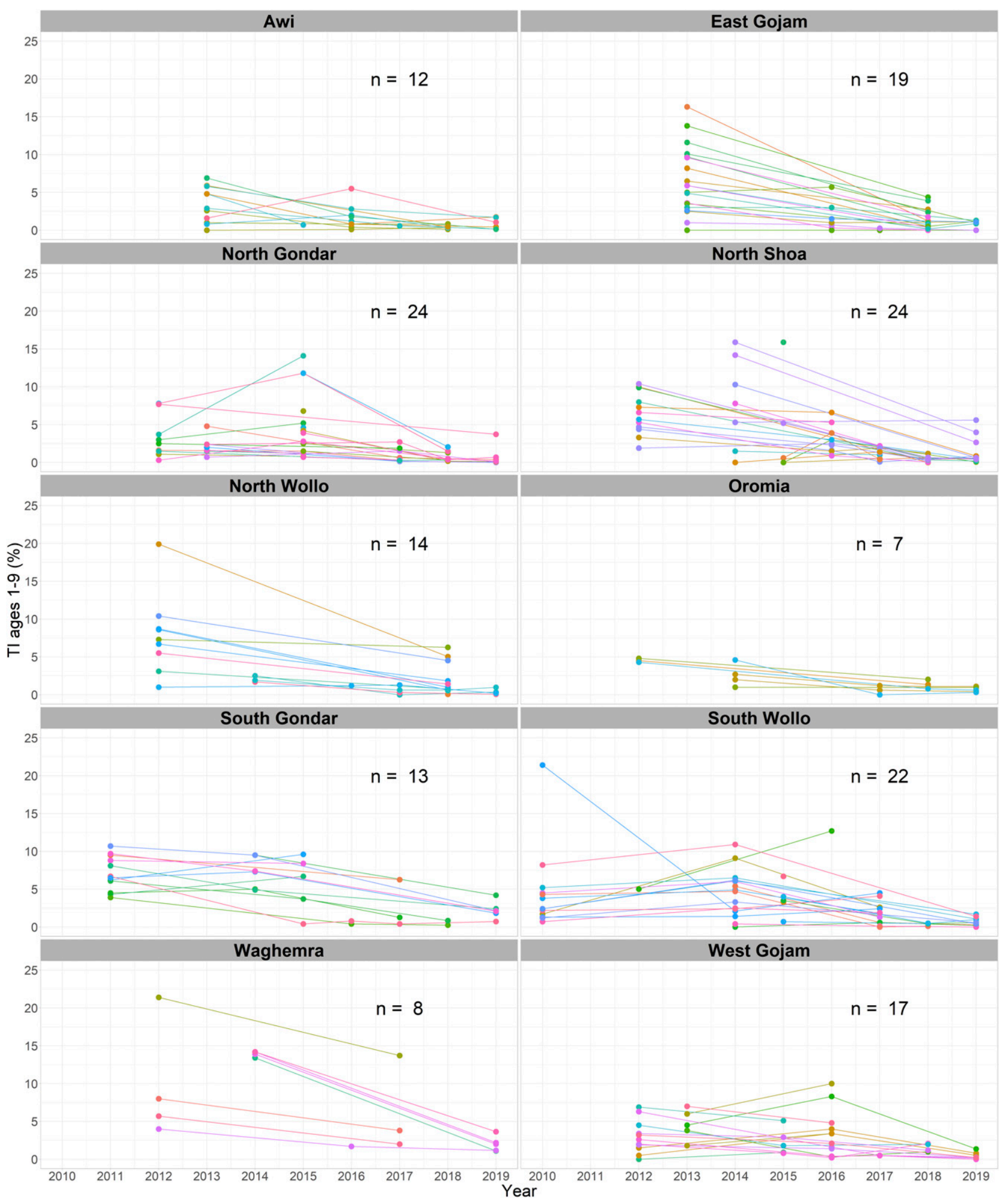

FIGURE 5. District-level trachomatous inflammation-intense (TI) prevalence over time among children aged 1-9 years by zone, Amhara, Ethiopia, 2010-2019 $(n=160)$. This figure appears in color at www.ajtmh.org.

elimination as a public health problem of $<5 \%$ TF. Across the age range, compared with most recent surveys, the prevalence of TF and TI were consistently lower than they were during the period of 2010-2015. A majority of districts had a very low prevalence of $\mathrm{TI}$, an indicator strongly correlated with
Ct infection, and $76 \%$ of districts that had completed surveillance surveys remained below the $5 \%$ TF threshold. ${ }^{16,29}$ Despite these successes, challenges remain for the region, as $19 \%$ of districts still have a TF prevalence $\geq 30 \%$ and will require more years of $A, F$, and $E$ interventions. If the global 


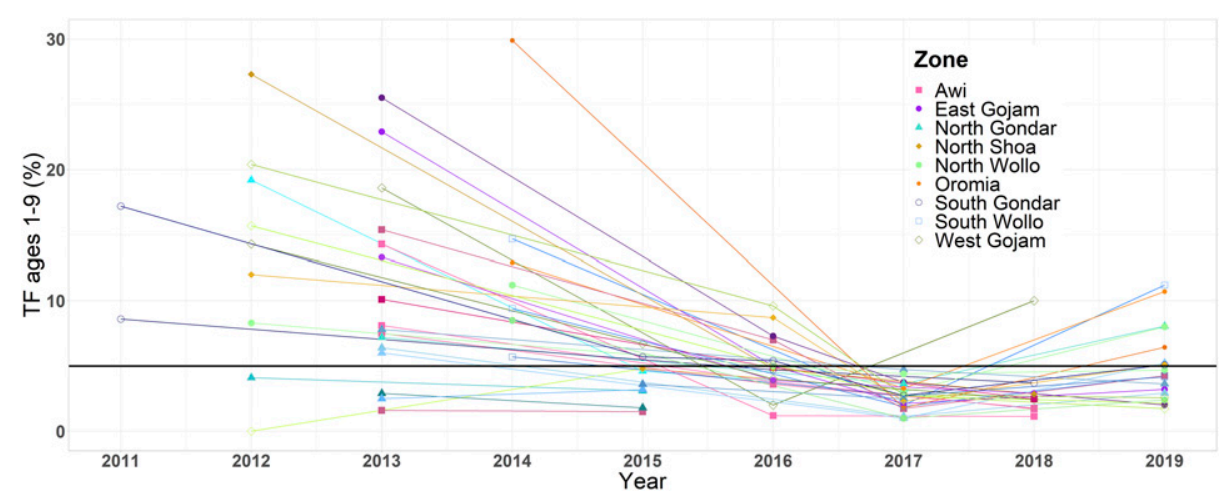

FIGURE 6. Longitudinal trends in the prevalence of Trachomatous inflammation-follicular (TF) among children aged 1-9years for those 37 districts which reached surveillance survey, Amhara, Ethiopia, 2011-2019. *Districts are grouped by their respective zone to aid in visualization. This figure appears in color at www.ajtmh.org.

trachoma program is to reach the trachoma elimination as a public health problem goal, regions like Amhara which contain areas of persistent trachoma are going to need significant enhancements to the $A, F$, and $E$ strategies.

After approximately 8-10 years of $A, F$, and $E$ interventions in Amhara, 30 districts remained trachoma hyperendemic
( $\geq 30 \% \mathrm{TF})$. Prior research in the region has demonstrated that by most measures available to programs (administrative, logbook audit, and self-report), mass drug administration (MDA) coverage has approached or been greater than $80 \%$, the minimum recommended level. ${ }^{19,20,30}$ Furthermore, results from whole genome sequencing of 99 samples collected

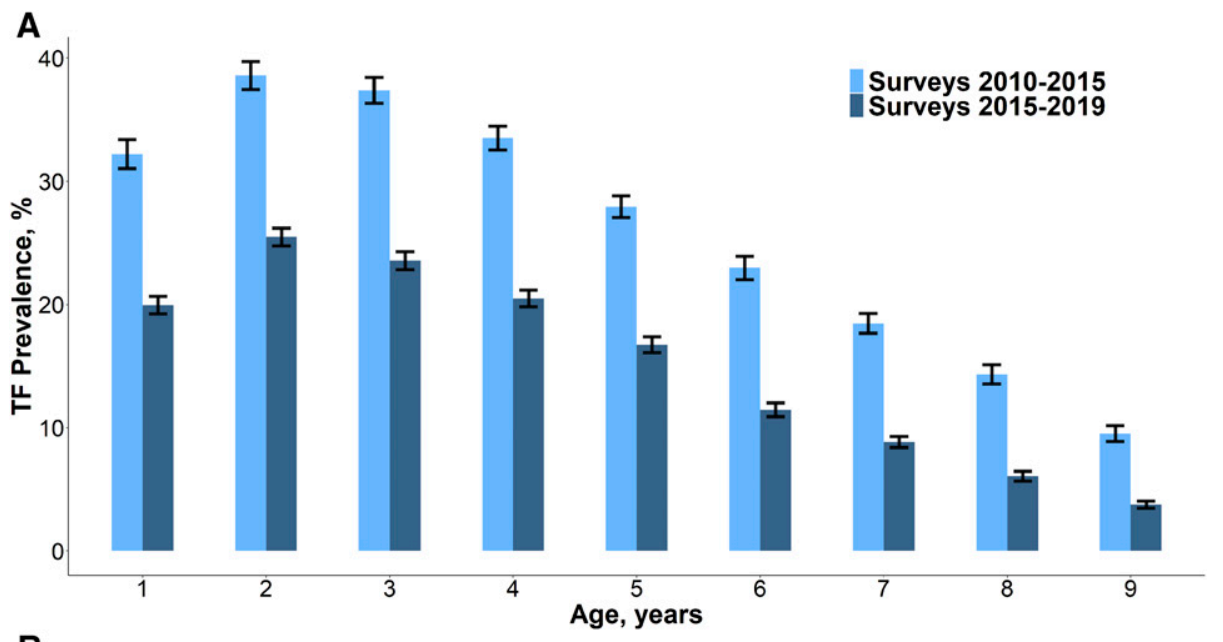

B

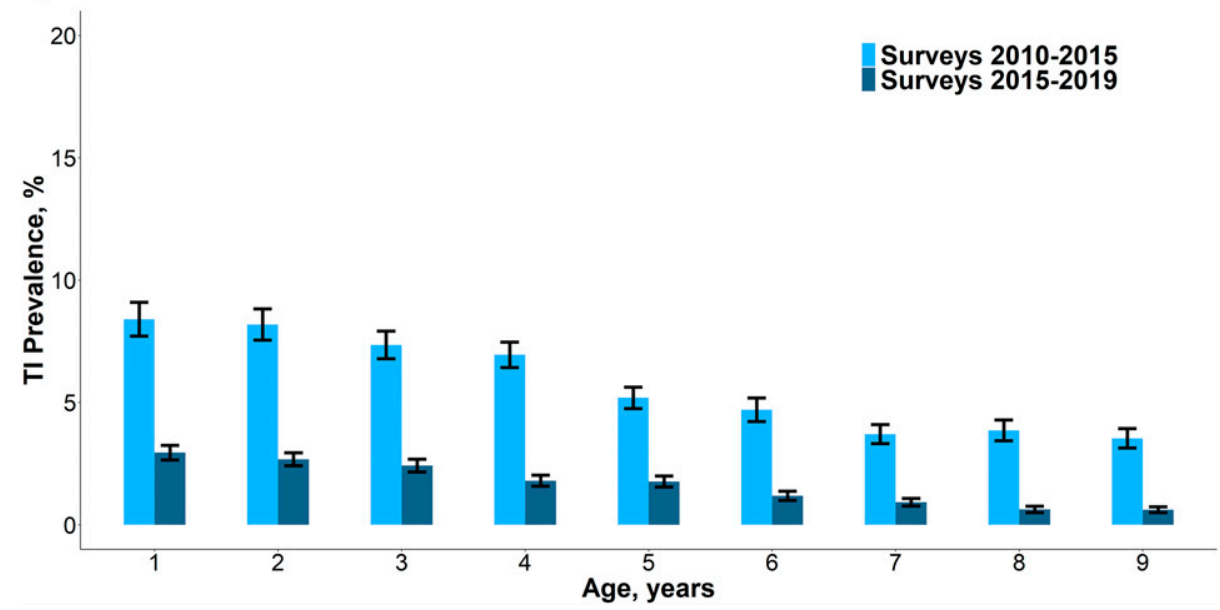

FIGURE 7. Age-specific prevalence of (A) trachomatous inflammation-follicular (TF) and (B) trachomatous inflammation-intense (TI) among children aged 1-9 years for surveys conducted as part of the first impact surveys (2010-2015) and for those conducted in the most recent round of surveys (2015-2019), Amhara, Ethiopia. This figure appears in color at www.ajtmh.org. 
throughout the region after five rounds of MDA failed to show any evidence of antimicrobial resistance in $\mathrm{Ct}^{31}$ Both modeling studies and randomized control trials have demonstrated that hyperendemic settings will likely take 10 or more years to reach elimination thresholds under current treatment guidelines of one round of MDA per year. ${ }^{32-34}$ In Amhara specifically, even two rounds of MDA per year for 8 years could not reduce TF prevalence less than $30 \%$ under randomized trial conditions. ${ }^{35}$ The persistent trachoma experienced in parts of the region may reflect a more severe trachoma epidemiological pattern not commonly observed in many parts of the world. ${ }^{17}$ Therefore, programs facing persistent trachoma must consider enhanced or targeted MDA strategies as soon as possible.

As per current WHO recommendations, once districts have reached the elimination threshold of $<5 \% \mathrm{TF}$, MDA ceases. After a period of at least 2 years without MDA, a surveillance survey is conducted to determine whether elimination has been sustained. In an earlier report from Amhara, it was demonstrated that the first five districts to reach the elimination threshold did maintain a $<5 \%$ TF prevalence at surveillance survey despite being geographically adjacent to endemic districts. ${ }^{23}$ This report extends those findings and shows that $76 \%$ of the 37 districts which have had surveillance surveys stayed below the threshold after ceasing MDA. There were, however, nine districts that had a rebound of prevalence of TF $\geq 5 \%$ at surveillance survey, and thus needed to restart their MDA program, a costly endeavor for programs. These nine districts had a TF prevalence that ranged between $5 \%$ and $11 \%$, with lower confidence limits that crossed the $5 \%$ threshold. It is difficult to determine whether a prevalence $\geq$ $5 \%$ at surveillance survey represents true recrudescence of trachoma transmission in these districts, or whether these results were due to sampling variability and stochastic variation in the data. ${ }^{36}$ The addition of $C t$ infection monitoring to surveillance surveys, measured by nucleic acid amplification tests or serology, may help to elucidate this issue. Given the programmatic costs of restarting MDA distribution, trachoma control programs might consider a wait-and-watch approach for those districts with a TF prevalence between $5 \%$ and $10 \%$ at surveillance survey. With this approach, programs would wait 12 months and repeat the surveillance survey to determine if restarting MDA is truly warranted.

Interventions focused on $F$ and $E$ will likely be important to sustaining elimination of trachoma as a public health problem. The control program in Amhara has a long history of supporting and advocating for latrine construction and intensive health education, and indeed improvements in water and sanitation indicators have been observed since the start of the program. $^{8,15}$ Data from surveys conducted through 2019 demonstrated a $62 \%$ median household prevalence of access to improved water sources, a 39\% median household prevalence of water access within 30 minutes, and a $70 \%$ median prevalence of children with clean faces. Continued improvements in these indicators are needed, as recent evidence has demonstrated that lack of water access and unclean faces were associated with trachoma hot spots within Amhara. ${ }^{37}$ As part of $F$ and $E$ interventions in the region, the program has partnered with educators to improve awareness of trachoma and trachoma preventive strategies as part of a region-wide STP. Trachoma programming in schools has been shown to improve health knowledge and facial cleanliness, although gains would likely be the greatest when programs are undertaken alongside improvements in environmental conditions. $^{21,38,39}$ In Waghemra, a zone with persistent trachoma and low water access, a community-randomized control trial is ongoing to test the effect of water and soap provision alongside school and village-based health education on reductions in $\mathrm{Ct}$ infection. ${ }^{40}$ Continued cross-sector collaboration by the WASH-NTD taskforce across Amhara should improve trachoma outcomes as well as reduce the burden of other hygiene-related diseases.

The data presented here were collected from surveys conducted over a 12-year period. Over this time, survey methodology and data collection platforms have evolved based on lessons learned and changes in WHO guidance, and some district boundaries have changed because of administrative decisions at the regional level. Furthermore, there is uncertainty around each prevalence estimate, and therefore direct comparisons of districts across time should be made with caution. However, in every survey included here, sampling was carried out to ensure unbiased population-based estimates, and a rigorous trachoma inter-grader rating was maintained over the 12 years. ${ }^{41}$ In the most recent surveys, data on clean face were collected using the note field of the data collection platform which may have led to increased nondifferential misclassification with this variable. The goal of trachoma monitoring is to observe whether a district's TF prevalence has reached $<5 \%$ among children aged $1-9$ years. These longitudinal data allow for the progress toward that threshold to be displayed at a district-to-district level.

The next 3-5 years will be very important for the Trachoma Program in Amhara if the elimination as a public health problem target of 2030 is to be achieved. The data from Amhara demonstrated that there was a strong relationship between the level of trachoma endemicity around the start of interventions and eventual achievement of elimination thresholds. Countries and regions with TF levels $\geq 30 \%$ near the start of programming could expect a considerably long elimination time line under current strategies. Within persistently hyperendemic districts, the program needs to test and then scaleup enhanced MDA strategies such as those targeting young children, the reservoir of the disease. ${ }^{42}$ The quarterly treatment of children has already been shown to be statistically significantly better than annual MDA in a cluster-randomized trial conducted in Amhara, and should be considered for further implementation, particularly for those districts persisting at $\geq 30 \%$ TF. ${ }^{43}$ Without changing the MDA strategy, it is possible that trachoma may still be endemic in these districts after another 10-20 years, an extremely costly proposition. Last, the program needs to continue its commitment to the $F$ and $E$ interventions and should look for ways to strengthen its partnerships with relevant sectors in the region. Although evidence of the efficacy of $F$ and $E$ interventions on trachoma outcomes is scarce, improvements in WASH would likely provide health benefits to the communities served by the program.

Before $A, F$, and $E$ interventions, the Amhara region was likely the most highly endemic region in the country with the highest burden of trachoma. Over the last 12 years, the Trachoma Control Program has made considerable progress toward reducing the burden of trachoma among children. With continued perseverance, strengthened partnerships, and innovative approaches, achieving elimination as a public health problem in Amhara is within reach. 
Received October 16, 2020. Accepted for publication November 23 , 2020.

Published online January 18, 2021.

Note: Supplemental table and figures appear at www.ajtmh.org.

Acknowledgments: We are grateful to all study participants who volunteered their time to participate in the study. We are also grateful for the collaboration with the Lions-Carter Center Sight-First Initiative.

Financial support: Funding was provided by many donors, including the Lions Clubs International Foundation, Noor Dubai Foundation, and in 2013 through the U.S. Agency for International Development ENVISION project led by RTI International.

Disclaimer: The authors' views expressed in this publication do not necessarily reflect the views of any funder, including the U.S. Agency for International Development or the U.S. government.

Authors' addresses: Eshetu Sata, Tigist Astale, Demelash Gessese, Zebene Ayele, Mulat Zerihun, Ambahun Chernet, Berhanu Melak, and Zerihun Tadesse, The Carter Center, Trachoma Control Program, Addis Ababa, Ethiopia, E-mails: eshetu.sata@cartercenter.org, tigist.astale @cartercenter.org, demelash.gessese@cartercenter.org, zebene.ayele@ cartercenter.org, mulat.zerihun@cartercenter.org, ambahun.chernet @cartercenter.org, berhanu.melak@cartercenter.org, and zerihun.tadesse@ cartercenter.org. Andrew W. Nute, Kimberly A. Jensen, Elizabeth Kelly Callahan, and Scott D. Nash, The Carter Center, Trachoma Control Program, Atlanta, GA, E-mails: andrew.nute@cartercenter.org, kim.jensen@ cartercenter.org, kelly.callahan@cartercenter.org, and scott.nash@ cartercenter.org. Mahteme Haile and Taye Zeru, Amhara Public Health Institute, Research and Technology Transfer Directorate, Bahir Dar, Ethiopia, E-mails: maykm24@yahoo.com and zerutaye@gmail.com. Melkamu Beyen and Adisu Abebe Dawed, Amhara Regional Health Bureau, Health Promotion and Disease Prevention, Bahir Dar, Ethiopia, E-mails: hpes14@gmail.com and adisua0@gmail.com. Fikre Seife, Federal Ministry of Health, Disease Prevention and Control Directorate, Addis Ababa, Ethiopia, E-mail: fikreseife5@gmail.com. Jeremiah Ngondi, RTI International, International Development (Global Health), London, United Kingdom, E-mail: jngondi@rti.org.

This is an open-access article distributed under the terms of the Creative Commons Attribution (CC-BY) License, which permits unrestricted use, distribution, and reproduction in any medium, provided the original author and source are credited.

\section{REFERENCES}

1. WHO, 2019. WHO alliance for the global elimination of trachoma by 2020: progress report on elimination of trachoma, 2018. Weekly Epidemiological Rec 94: 317-328.

2. WHO, 1998. WHA51.11 Global Elimination of Blinding Trachoma. Geneva, Switzerland: World Health Organization. Available at: http://www.who.int/neglected_diseases/mediacentre/WHA_ 51.11_Eng.pdf. Accessed September 15, 2020.

3. Cerulli LCC, Culasso F, Martelli M, Tria M, 1981. Epidemiolgoical study of trachoma in two regions of Ethiopia. Rev Int Trach Path 68: 147-155.

4. Okubagzhi G, 1997. Epidemiology of trachoma in north-western Ethiopia. East Afr Med J 64: 611-616.

5. Assefa TAD, Foster A, Schwrtz E, 2001. Results of trachoma rapid assessment in 11 villages of south Gondar zone, Ethiopia. Trop Doctor 31: 202-204.

6. Cumberland P, Hailu G, Todd J, 2005. Active trachoma in children aged three to nine years in rural communities in Ethiopia: prevalence, indicators and risk factors. Trans $R$ Soc Trop Med Hyg 99: 120-127.

7. Berhane Y, Worku A, 2006. National Survey on Blindness, Low Vision and Trachoma in Ethiopia. Addis Ababa, Ethiopia: Federal Ministry of Health of Ethiopia.

8. Ngondi $\mathrm{J}$ et al., 2009. Evaluation of three years of the SAFE strategy (surgery, antibiotics, facial cleanliness and environmental improvement) for trachoma control in five districts of Ethiopia hyperendemic for trachoma. Trans $R$ Soc Trop Med Hyg 103: 1001-1010.

9. Keenan JD, Lakew T, Alemayehu W, Melese M, House JI, Acharya NR, Porco TC, Gaynor BD, Lietman TM, 2011. Slow resolution of clinically active trachoma following successful mass antibiotic treatments. Arch Ophthalmol 129: 512-513.

10. Gebre T, Ayele B, Zerihun M, Genet A, Stoller NE, Zhou Z, House JI, Sun NY, Ray KJ, Emerson PM, 2012. Comparison of annual versus twice-yearly mass azithromycin treatment for hyperendemic trachoma in Ethiopia: a cluster-randomised trial. Lancet 379: 143-151.

11. Emerson PM et al., 2008. Integrating an NTD with one of "The big three": combined malaria and trachoma survey in Amhara region of Ethiopia. PLoS Negl Trop Dis 2: e197.

12. Ngondi J, Teferi T, Gebre T, Shargie EB, Zerihun M, Ayele B, Adamu L, King JD, Cromwell EA, Emerson PM, 2010. Effect of a community intervention with pit latrines in five districts of Amhara, Ethiopia. Trop Med Int Health 15: 592-599.

13. King JD et al., 2013. Intestinal parasite prevalence in an area of Ethiopia after implementing the SAFE strategy, enhanced outreach services, and health extension program. PLoS Negl Trop Dis 7: e2223.

14. King JD et al., 2014. Prevalence of trachoma at sub-district level in Ethiopia: determining when to stop mass azithromycin distribution. PLoS Negl Trop Dis 8: e2732.

15. Stewart AEP et al., 2019. Progress to eliminate trachoma as a public health problem in Amhara national regional state, Ethiopia: results of 152 population-based surveys. Am J Trop Med Hyg 101: 1286-1295.

16. Nash SD et al., 2018. Ocular Chlamydia trachomatis infection under the SAFE strategy in Amhara, Ethiopia, 2011-2015. Clin Infect Dis 67: 1840-1846.

17. Oldenburg CE, 2019. One size does not fit all: achieving trachoma control by 2030. Am J Trop Med Hyg 101: 1189-1190.

18. Nash SD et al., 2020. Ocular Chlamydia trachomatis infection and infectious load among pre-school aged children within trachoma hyperendemic districts receiving the SAFE strategy, Amhara region, Ethiopia. PLoS Negl Trop Dis 14: e0008226.

19. Astale T et al., 2018. Population-based coverage survey results following the mass drug administration of azithromycin for the treatment of trachoma in Amhara, Ethiopia. PLoS Negl Trop Dis 12: e0006270.

20. Ebert CD et al., 2019. Population coverage and factors associated with participation following a mass drug administration of azithromycin for trachoma elimination in Amhara, Ethiopia. Trop Med Int Health 24: 493-501.

21. Sata E et al., 2018. Evaluation of a school trachoma health program after one year of program implementation in primary schools of Amhara regional state, Ethiopia. Conference abstract, New Orleans, LA. Am Soc Trop Med Hyg.

22. WHO, 2010. Report of the 3rd Global Scientific Meeting on Trachoma. Baltimore, MD, World Health Organization.

23. Nash SD et al., 2018. Trachoma prevalence remains below threshold in five districts after stopping mass drug administration: results of five surveillance surveys within a hyperendemic setting in Amhara, Ethiopia. Trans $R$ Soc Trop Med Hyg 112: 538-545.

24. Solomon AW et al., 2015. The global trachoma mapping project: methodology of a 34-country population-based study. Ophthalmic Epidemiol 22: 214-225.

25. King JD et al., 2013. A novel electronic data collection system for largescale surveys of neglected tropical diseases. PLoS One 8: e74570.

26. WHO/UNICEF, 2016. Improved and unimproved water sources and sanitation facilities. Joint Monitoring Programme for Water Supply, Sanitation and Hygiene (JMP). Geneva, Switzerland: World Health Organization.

27. Thylefors B, Dawson CR, Jones BR, West SK, Taylor HR, 1987. A simple system for the assessment of trachoma and its complications. Bull World Health Organ 65: 477-483.

28. Lumley T, 2004. Analysis of complex survey samples. J Stat Softw 9: 1-19.

29. Ramadhani AM, Derrick T, Macleod D, Holland MJ, Burton MJ, 2016. The relationship between active trachoma and ocular Chlamydia trachomatis infection before and after mass antibiotic treatment. PLoS Negl Trop Dis 10: e0005080.

30. Jordan AM et al., 2017. Village-level medicaiton logbook audit following a trachoma mass drug administration campaign in Amahara, Ethiopia in 2016. Conference abstract, Baltimore, MD. Am Soc Trop Med Hyg. 
31. Pickering $\mathrm{H}$ et al., 2020. Genomics of ocular chlamyda trachomatis after 5 years of SAFE interventions for trachoma in Amhara, Ethiopia. J Infect Dis Oct 9: jiaa615.

32. West SK, Munoz B, Mkocha H, Gaydos CA, Quinn TC, 2011. Number of years of annual mass treatment with azithromycin needed to control trachoma in hyper-endemic communities in Tanzania. J Infect Dis 204: 268-273.

33. Pinsent A, Burton MJ, Gambhir M, 2016. Enhanced antibiotic distribution strategies and the potential impact of facial cleanliness and environmental improvements for the sustained control of trachoma: a modelling study. BMC Med 14: 71.

34. Borlase A, Blumberg S, Callahan EK, Diener MS, Nash SD, Porco TC, Solomon AW, Lietman TM, Prada JM, Hollingsworth D, 2020. Modelling trachoma post 2020: opportunities for mitigating the impact of COVID-19 and accelerating progress towards elimination. Trans R Soc Trop Med Hyg. (In Press).

35. Keenan JD et al., 2018. Mass azithromycin distribution for hyperendemic trachoma following a cluster-randomized trial: a continuation study of randomly reassigned subclusters (TANA II). PLoS Med 15: e1002633.

36. Godwin W et al., 2020. Trachoma prevalence after discontinuation of mass azithromycin distribution. J Infect Dis 221: S519-S524.
37. Altherr FM et al., 2019. Associations between water, sanitation and hygiene (WASH) and trachoma clustering at aggregate spatial scales, Amhara, Ethiopia. Parasit Vectors 12: 540.

38. Lewallen S, Massae P, Tharaney M, Somba M, Geneau R, Macarthur C, Courtright P, 2008. Evaluating a school-based trachoma curriculum in Tanzania. Health Educ Res 23: 1068-1073.

39. Dickman L, Melek B, 2013. Children as agents of change in trachoma control. Community Eye Health 26: 18.

40. Aiemjoy K et al., 2018. Defining diarrhea: a population-based validation study of caregiver-reported stool consistency in the Amhara region of Ethiopia. Am J Trop Med Hyg 98: 1013-1020.

41. Nute AW et al., 2018. Trachoma grader reliability results from nine trachoma impact survey trainings conducted in Amhara, Ethiopia, 2011-2017. Conference abstract, New Orleans, LA. Am Soc Trop Med Hyg.

42. Schachter $\mathrm{J}$ et al., 1999. Azithromycin in control of trachoma. Lancet 354: 630-635.

43. House $\mathrm{Jl}$ et al., 2009. Assessment of herd protection against trachoma due to repeated mass antibiotic distributions: a cluster-randomised trial. Lancet 373: 1111-1118. 